Title: Europium Cyclooctatetraene Nanowire Carpets: A LowDimensional, Organometallic, and Ferromagnetic Insulator $\begin{array}{ll}\text { Author(s): } & \text { Huttmann, F., Rothenbach, N., Kraus, S., Ollefs, K., Arruda, L. M., Bernien, M., ... } \\ \text { Wende, H. }\end{array}$

Document type: Postprint

Terms of Use: $\quad$ Copyright applies. A non-exclusive, non-transferable and limited right to use is granted. This document is intended solely for personal, non-commercial use.

Citation:

Huttmann, F., Rothenbach, N., Kraus, S., Ollefs, K., Arruda, L. M., Bernien, M., ... Wende, H. (2019). Europium Cyclooctatetraene Nanowire Carpets: A Low-Dimensional, Organometallic, and Ferromagnetic Insulator. The Journal of Physical Chemistry Letters, 10(5), 911-917. https://doi.org/10.1021/acs.jpclett.8b03711

This document is the Accepted Manuscript version of a Published Work that appeared in final form in The Journal of Physical Chemistry Letters, copyright $\odot$ American Chemical Society after peer review and technical editing by the publisher. To access the final edited and published work see http://dx.doi.org/10.1021/acs.jpclett.8b03711. 


\title{
Europium Cyclooctatetraene Nanowire Carpets: A Low-dimensional, Organometallic, and Ferromagnetic Insulator
}

\author{
Felix Huttmann, ${ }^{\dagger}$ Nico Rothenbach, ${ }^{\ddagger}$ Stefan Kraus, ${ }^{\dagger}$ Katharina Ollefs, $^{\ddagger}$ \\ Lucas M. Arruda," Matthias Bernien, "Danny Thonig, ${ }^{\S}$ Anna Delin, ${ }^{\S}, \|, \perp$ Jonas \\ Fransson, ${ }^{\S}$ Kurt Kummer,"\# Nicholas B. Brookes,\# Olle Eriksson, $\$$,@ Wolfgang \\ Kuch, "Thomas Michely, ${ }^{\dagger}$ and Heiko Wende*, \\ †II. Physikalisches Institut, Universität zu Köln, Zülpicher Strasse 77, D-50937 Köln, \\ Germany \\ †Universität Duisburg-Essen and Center for Nanointegration Duisburg-Essen (CENIDE), \\ Lotharstr. 1, D-47057 Duisburg, Germany \\ 【Institut für Experimentalphysik, Freie Universität Berlin, Arnimallee 14, 14195 Berlin, \\ Germany \\ $\S$ Department of Physics and Astronomy, Materials Theory, Uppsala University, SE-75120 \\ Uppsala, Sweden \\ ||Department of Applied Physics, School of Engineering Sciences, KTH Royal Institute of \\ Technology, Electrum 229, SE-16440 Kista, Sweden \\ $\perp$ SeRC (Swedish e-Science Research Center), KTH Royal Institute of Technology, \\ SE-10044 Stockholm, Sweden \\ \# European Synchrotron Radiation Facility, 71 Avenue des Martyrs, CS40220, F-38043 \\ Grenoble Cedex 9, France \\ @ School of Science and Technology, Örebro University, SE-701 82 Örebro, Sweden \\ E-mail: heiko.wende@uni-due.de
}

We investigate the magnetic and elec- 17 tronic properties of europium cyclooctate- 18 traene nanowires (EuCot) by means of low- 19 temperature x-ray magnetic circular dichroism 20 (XMCD) and scanning tunneling microscopy 21 (STM) and spectroscopy (STS). The EuCot 22 nanowires are prepared in situ on a graphene 23 surface. STS measurements identify EuCot as 24 a wide-band-gap semiconductor with a band 25 gap of $2.3 \mathrm{eV}$. By means of $\mathrm{Eu} M_{5,4}$ edge $\mathrm{XMCD}$, orbital and spin magnetic moments of $(-0.1 \pm 0.3) \mu_{B}$ and $(+7.0 \pm 0.6) \mu_{B}$, respectively, were determined. Field-dependent measurements of the XMCD signal at the $\mathrm{Eu} M_{5}$ edge show hysteresis for grazing $\mathrm{x}$-ray incidence at $5 \mathrm{~K}$, thus confirming EuCot as a ferromag- netic material. Our density functional theory calculations reproduce the experimentally observed bandgap. Modelling the experimental results theoretically, we find that the effective interatomic exchange interaction between $\mathrm{Eu}$ atoms is of the order of meV, that magnetocrystalline anisotropy energy is roughly half as big and that dipolar energy is approximately ten times lower. 
TOC ENTRY REQUIRED
Sandwich molecular wires (SMWs) are a particular one-dimensional class of organometallic structures, distinct from zero-dimenensional molecular magnets, ${ }^{1,2}$ two-dimensional organometallic networks ${ }^{3-5}$ and molecular magnetic hybrid structures on surfaces. ${ }^{6,7}$ They consist of a periodic sequence of $4 f$ rare-earth metal cations, predominantly ionically bound and eightfold coordinated to planar aromatic anions, based on the cyclooctatetraene $\left(\mathrm{C}_{8} \mathrm{H}_{8}\right.$, briefly Cot $)$ molecule as ligand. ${ }^{8}$ Due to organometallic hybridization between the metal atomic states and the extended $\pi$ orbitals of the Cot, the metal ions in the wire were proposed to couple magnetically. ${ }^{9}$ These systems could be more stable magnetic units than single-molecule magnets, and could display larger magnetic anisotropy with correspondingly higher blocking temperatures.

A prime example is the EuCot SMW, for which chain lengths of up to 30 formula units could be achieved by Hosoya et al. through gas phase synthesis. ${ }^{10}$ Liquid phase synthesis was realized by Tsuji et al, ${ }^{11}$ though the product was contaminated with ferromagnetic EuO, making the interpretation of its magnetic properties problematic. Recently some of us introduced an on-surface synthesis method for EuCot, which operates under ultra-high vacuum conditions and yields a clean, phase-pure product with wire lengths up to 1000 formula units. $^{12}$

In Stern-Gerlach type experiments by Miyajima et al. ${ }^{13}$ the magnetic moment of EuCot was found to increase linearly with chain length and to be consistent with $m=7 \mu_{\mathrm{B}}$ for each $\mathrm{Eu}$ ion, as expected for $\mathrm{Eu}^{2+}$. Though experiments up to now could not make a statement on the presence of magnetic coupling between the paramagnetic $\mathrm{Eu}^{2+}$ ions in EuCot, in density functional theory (DFT) calculations a ferromagnetic coupling of the $\mathrm{Eu}^{2+}$ ions with $m=7 \mu_{\mathrm{B}}$ is invariably found. ${ }^{9,14-16}$ For the infinite wire, the ferromagnetic state is favored over the antiferromagnetic state by $2.5 \mathrm{meV}$ according to Atodiresei et al., ${ }^{9}$ by $6 \mathrm{meV}$ in the calculations of $\mathrm{Xu}$ et al., ${ }^{15}$ and by $1.2 \mathrm{meV}$ in theoretical work of Yao et al. ${ }^{16}$ EuCot is semiconducting with electronic band gaps of $2.0 \mathrm{eV}$ 
resp. $1.92 \mathrm{eV}$ for the majority channel and of 126 $3.1 \mathrm{eV}$ resp. $2.94 \mathrm{eV}$ for the minority chan- ${ }_{127}$ nel as found in the DFT calculations of $\mathrm{Xu}$ et ${ }_{128}$ al. ${ }^{15}$ resp. Yao et al. ${ }^{16}$ Furthermore, EuCot ${ }_{129}$ wires suspended between $\mathrm{Au}$ electrodes were 130 proposed to be nearly perfect spin filters by $\mathrm{Xu} 131$ et al. ${ }^{15}$

Based on the new on-surface synthesis 133 method for EuCot, in this contribution we 134 investigate the magnetic and electronic proper- 135 ties of EuCot SMWs experimentally by using 136 element-specific low-temperature x-ray mag- 137 netic circular dichroism (XMCD) experiments 138 and scanning tunneling spectroscopy (STS). We 139 experimentally confirm the theoretical propo- 140 sition of EuCot being a ferromagnetic semi- 14 conductor. At $5 \mathrm{~K}$ we find an open hysteresis 142 loop for magnetization along the wire axis and 143 considerable magnetic anisotropy by angular- ${ }_{144}$ and field-dependent XMCD investigations. $\quad{ }_{145}$

Scanning tunneling microscopy (STM), STS, 146 and low-energy electron diffraction (LEED) ${ }_{147}$ measurements were conducted in the STM ${ }_{148}$ lab in Cologne, while x-ray absorption spec- 149 troscopy (XAS) and XMCD measurements 150 complemented by sample characterization with 151 STM and micro-channel plate (MCP) LEED ${ }_{152}$ were conducted at the high-field-magnet end ${ }_{153}$ station of the ID32 beamline of the European 154 Synchrotron Radiation Facility (ESRF).

155

Prior to each experiment, the $\operatorname{Ir}(111)$ sam- 156 ple was prepared by cycles of noble gas sput- ${ }_{157}$ tering (Xe or Ar), flash annealing to $1500 \mathrm{~K} 158$ (Cologne) or $1670 \mathrm{~K}$ (ESRF). At the ESRF, ini- 159 tial oxygen firing at temperatures up to $1670 \mathrm{~K}{ }_{160}$ was applied, too. A fully closed, well-oriented ${ }_{161}$ layer graphene $(\mathrm{Gr})$ was prepared by room- 162 temperature ethylene adsorption until satura- 163 tion, thermal decomposition at $1470 \mathrm{~K}$ and sub- 164 sequent high-temperature exposure at $1270 \mathrm{~K}_{165}$ to $1 \times 10^{-6}$ mbar ethylene. ${ }^{17}$ The same quality 166 Gr sheet was realized at the ESRF through ex- 167 posure to $1 \times 10^{-6}$ mbar ethylene for $600 \mathrm{~s}$ at 168 a sample temperature of $1500 \mathrm{~K}$. The orienta- 169 tion and closure of the Gr layer was confirmed 170 in both labs through LEED and STM. A $60 \% 171$ coverage of $\mathrm{Gr} / \mathrm{Ir}(111)$ with EuCot nanowire 172 islands of single-layer height was realized by 173 simultaneous room-temperature exposure to a 174 pressure of $5 \times 10^{-7}$ mbar Cot molecules and a flux of $1.9 \times 10^{16} \mathrm{~s}^{-1} \mathrm{~m}^{-2} \mathrm{Eu}$ atoms for $105 \mathrm{~s}$. Cot molecules were admitted through a gas dosing valve and Eu was sublimated from a watercooled Knudsen cell.

The magnetic properties of the Eu ions, in the EuCot nanowires, were investigated by means of XAS- and XMCD-measurements. The data were taken in drain current mode using fully $(100 \%)$ circularly polarized light. The magnetic field of up to $9 \mathrm{~T}$ was aligned either parallel or antiparallel to the incident x-ray beam. By rotating the sample around the vertical axis we could adjust the angle $\theta$ of the incident $\mathrm{x}$-rays to the surface normal between normal incidence $\left(\theta=0^{\circ}\right)$ and grazing incidence $\left(\theta=60^{\circ}\right)$. Hence, we have been able to obtain information about the magnetic anisotropy of the nanowires. The sample can reach temperatures down to $\sim 5 \mathrm{~K}$ and can go up to $\sim 325 \mathrm{~K}$. To avoid nonmagnetic artifacts due to switching either the magnetic field or the polarization of the x-rays, all magnetic measurements have been done for all four combinations of field direction and polarization. In order to minimize radiation damage of the $\mathrm{Eu}$ Cot wires, we applied settings of the mirrors that yield a defocusing of $1 \mathrm{~mm}$ in vertical direction, while the beam size in horizontal direction was about $100 \mu \mathrm{m}$. Using these settings, subsequently recorded XAS and field-dependent XMCD magnetization curves did not show significant changes with time. We performed the magnetic measurements on several identically prepared samples to ensure that the total exposition time of our samples to the x-rays is minimized.

The electronic structure calculations employed the full-potential linear muffin-tin RSPt code. ${ }^{18}$ The calculations made use of the generalized gradient approximation and the basis set consisted of spd basis functions while the $4 f$ states were treated as non-hybridizing core states. The $4 f$ shell was allowed to spinpolarize forming a net spin moment of $7 \mu_{B}$, while the orbital moment in accordance to Russel-Saunders coupling is zero. The polarization of the $4 f$ shell induced a spin-polarization also of the itinerant valence electrons, via the 
exchange-correlation functional. The calcula- 213 tions ignored the influence of the substrate and ${ }_{214}$ focused only on the free EuCot molecule. Also, 215 the first principles calculations were performed 216 only for the electronic structure, magnetic mo- 217 ments and valence stability.

The thermal ground state of atomistic spins 219 $\left\{\vec{m}_{i}\right\}=m_{i}\left\{\vec{e}_{i}\right\}$ at site $i$ in the Eu wire is ob- 220 tained from energy minimization by Monte 221 Carlo simulations on the Metropolis algo- 222 rithm. ${ }^{19}$ The Hamiltonian is

$$
\begin{aligned}
\mathcal{H} & =-\sum_{<i, j>} J \vec{m}_{i} \cdot \vec{m}_{j}-\sum_{i j} \vec{m}_{i} \mathbf{Q}_{i j} \vec{m}_{j} \\
& +K \sum_{i}\left(\vec{m}_{i} \cdot \hat{e}\right)^{2}-\mu_{B} \vec{B} \sum_{i} \vec{m}_{i}
\end{aligned}
$$

consisting of Heisenberg interaction between ${ }_{230}^{229}$ nearest-neighbor spins of strength $J$, dipole- ${ }_{231}^{230}$ dipole interaction via the dipolar tensor $\mathbf{Q}_{i j}$ in ${ }_{232}^{231}$ the point-dipol approximation, ${ }^{20}$ uniaxial mag- ${ }_{233}$ netocrystaline anisotropy and Zeeman term, respectively. $K$ is the anisotropy constant and $\vec{B}_{235}^{234}$ the external magnetic field.

With knowledge about the exchange cou- ${ }^{236}$ plings $J$, one can estimate the phase transi- ${ }^{237}$ tion temperature $T_{C}$ from mean field theory ${ }_{239}$ via $k_{B} T_{C}^{M F}=32 \sum_{j} J_{0 j}$ or from Monte Carlo ${ }_{240}^{239}$ simulations via both the susceptibility $\chi$ and ${ }_{241}^{240}$ Binder's fourth cumulant ${ }^{19}$ for different simulated system sizes. By definition, ${ }^{21}$ the phase ${ }_{243}^{242}$ transition temperature $T_{C}$ is determined only ${ }_{244}^{243}$ from interaction terms in Eq. (1); $K$ and $B$ are ${ }_{245}^{244}$ zero. By varying $B$ along the wire, we obtain ${ }^{245}$ hystereses loops, where the coercive field $B^{\text {coer }}{ }^{246}$ is extracted from an interpolation of the aver- ${ }^{247}$ age magnetization as a function of the external ${ }_{249}^{248}$ magnetic field $M(B)$ and $M\left(B^{\text {coer }}\right)=0$. The ${ }_{250}{ }^{249}$ Monte Carlo simulations were performed using ${ }_{251}$ the UppASD software. ${ }^{22,23}$

We simulate a repetition (10 times) of a $\mathrm{Eu}^{252}$ wire consisting of 1000 atoms without periodic ${ }_{254}$ boundary condition to form a carpet. To re- ${ }^{254}$ duce thermal noise we account for 15 replica of ${ }_{256}^{255}$ this setup. It turned out that this size is still to ${ }_{257}$ small to see domain wall nucleation, although ${ }_{258}{ }^{257}$ it is allowed by the model in Eq. (1). The easy ${ }_{259}^{258}$ axis $\vec{e}$ is in-plane and along the wires. $J, K_{260}^{259}$ as well as temperature $T$ are parameters of our ${ }_{261}^{260}$ study. Note that the nearest neighbor interaction $J$ is only along the wire; direct exchange between the wires is set to zero.

Fig. 1 depicts the structure of the EuCot nanowire film investigated by STS, XAS, and XMCD. As visible in Fig. 1(a), the film with a coverage of $0.6 \mathrm{ML}$ is formed. The analysis shows that the islands are $(6.1 \pm 0.5) \AA$ high with straight edges that consist of parallel nanowires. The islands are distributed in almost random orientation on $\mathrm{Gr} / \mathrm{Ir}(111)$. Each wire is composed of an alternating sequence of $\mathrm{Eu}^{2+}$ cations and $\mathrm{Cot}^{2-}$ anions [compare Fig. 1(b)]. ${ }^{10,24}$ Each $\mathrm{Eu}^{2+}$ is eightfold coordinated (hapticity $\eta=8$ ) to neighboring $\mathrm{Cot}^{2-}$. Based on STM and LEED, we find primitive translations of the wire carpet unit cell (light blue in the inset of Fig. 1(a) and in Fig. 1(c)) of $4.4 \AA$ and $7.15 \AA$. These values correspond to an intra-wire separation of $4.4 \AA$ and an inter-wire separation of $6.8 \AA$. The $42 \mathrm{eV}$ MCP-LEED pattern in Fig. 1(d) displays first-order moiré reflections around the $(0,0)$ spot and two concentric diffraction rings characteristic for the EuCot primitive translations defined by the EuCot carpets and consistent with a close-to random orientation distribution of the EuCot islands. A slight preference for island orientations along $\langle 1 \overline{1} 0\rangle$ and $\langle 11 \overline{2}\rangle$ is visible in the MCP-LEED pattern through intensity variations of the diffraction rings. Due to the low electron beam currents, the EuCot MCP-LEED patterns were stable on the time scale of $1000 \mathrm{~s}$ and did not show degradation, while the EuCot reflections faded away on the time scale of $100 \mathrm{~s}$ in standard LEED.

We now turn to the discussion of the XAS and XMCD spectra. Since the magnetism of Eu originates from the $4 f$ electrons, we investigate the $\mathrm{Eu} M_{5,4}$ edges, i.e., transitions from initial $3 d_{5 / 2}$ and $3 d_{3 / 2}$ to the final $4 f$ states. Fig. 2(a) shows the XAS signal across these edges for a 0.6 ML EuCot coverage on $\mathrm{Gr} / \operatorname{Ir}(111)$. The measurements were performed at a sample temperature of $T=5 \mathrm{~K}$ and an external magnetic field of $B=9 \mathrm{~T}$ with left $\left(\mu^{-}\right)$and right $\left(\mu^{+}\right)$ circularly polarized x-rays at grazing incidence $\left(\theta=60^{\circ}\right)$ with respect to the sample surface. The spectra are presented on a vertical scale 

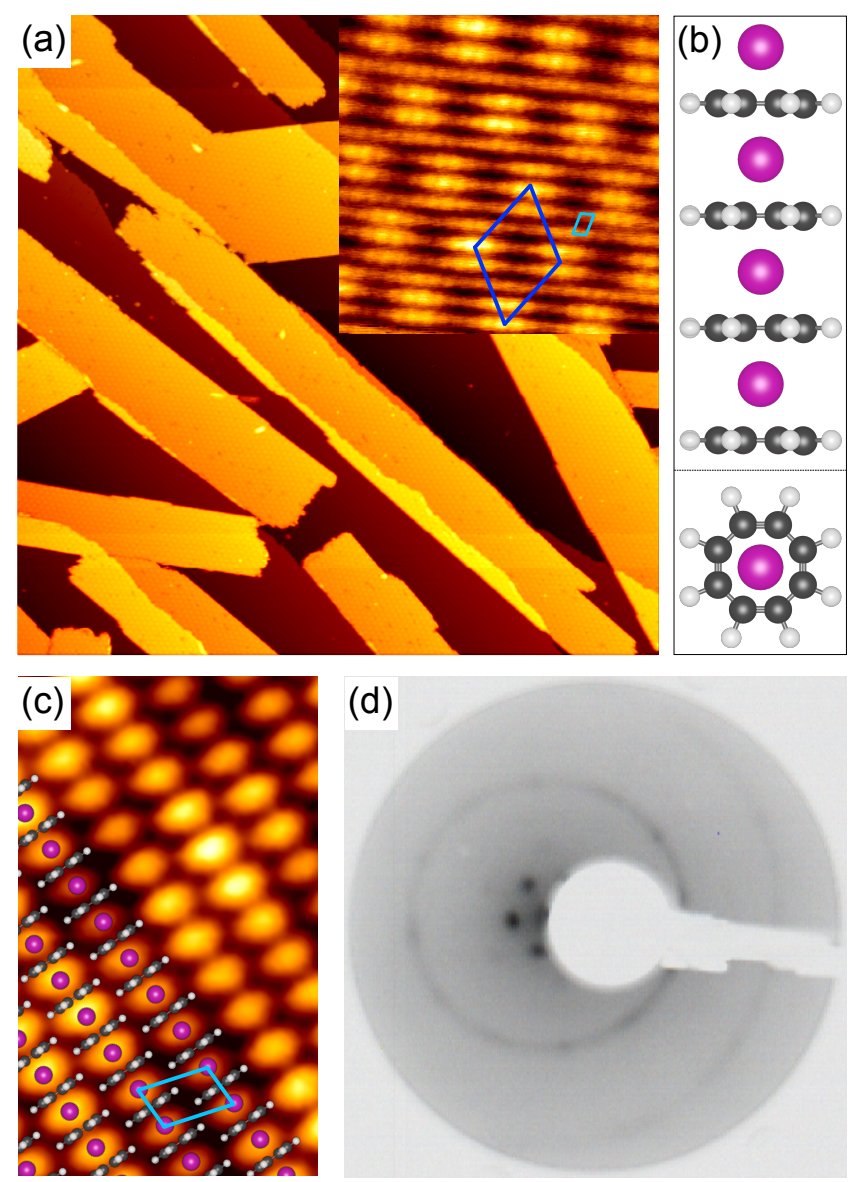

(d)

Figure 1: (a) STM topograph of a 0.6 ML EuCot nanowire film on graphene on $\operatorname{Ir}(111)$. Image size $260 \times 260 \mathrm{~nm}^{2}$, tunneling voltage $U=$ $-3.0 \mathrm{~V}$, tunneling current $I=52 \mathrm{pA}$. In the inset the wire structure can be identified clearly. The unit cell of the wire carpet is indicated as light blue rhomboid. Hexagonal pattern of height modulations is due to the $\mathrm{Gr} / \mathrm{Ir}(111)$ moiré. The moiré unit cell is indicated as a dark blue rhombus and has an edge length of $2.53 \mathrm{~nm}$. Image size $10 \times 10 \mathrm{~nm}^{2}, U=-3.0 \mathrm{~V}$, $I=58 \mathrm{pA}$. (b) Side view and view along the wire axis of the DFT-based structure model ${ }^{12}$ (Eu pink, C dark grey, $\mathrm{H}$ light grey). High-resolution topograph of EuCot, partially overlaid with a structural model and indicating experimentally measured geometry. The light blue rhombus indicates the wire carpet unit cell. Image size $5 \times 3 \mathrm{~nm}^{2}, U=-3.1 \mathrm{~V}, I=60 \mathrm{pA}$. (d) $42 \mathrm{eV}$ micro-channel plate LEED pattern of $0.6 \mathrm{ML}$ EuCot film on $\mathrm{Gr} / \operatorname{Ir}(111)$ after the film was used for the field-dependent XMCD magnetization curve measurements shown in Fig. 3. Two diffraction rings due to EuCot islands and moiré reflections around the $(0,0)$ spot are present.

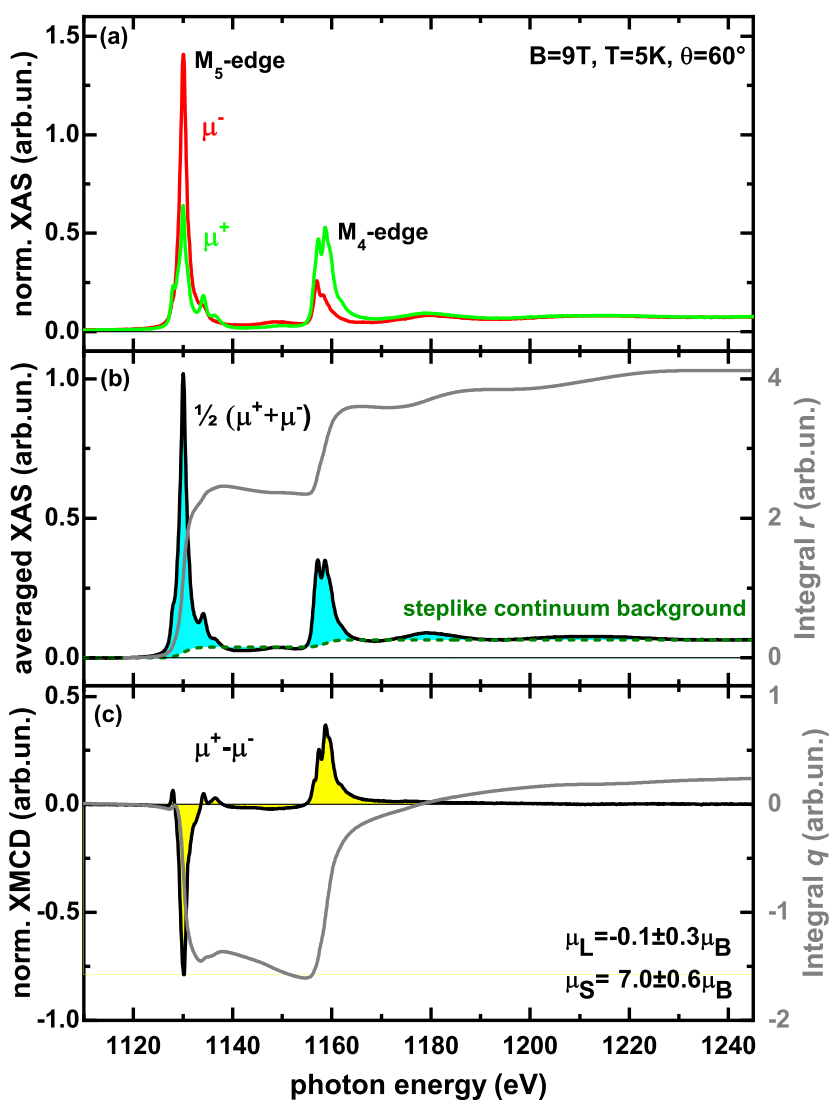

Figure 2: (a) XAS of the Eu $M_{5}$ and $M_{4}$ edges measured at grazing incidence with $\theta=60^{\circ}$, $T=5 \mathrm{~K}, B=9 \mathrm{~T}$, and with left $\left(\mu^{-}\right)$(red solid line) and right $\left(\mu^{+}\right)$(green solid line) circularly polarized x-rays. (b) Polarizationaveraged XAS $12\left(\mu^{+}+\mu^{-}\right)$(black solid line) with a step function (green dashed line) used to separate the $M_{5,4}$ contributions (blue area) from the continuum. Also indicated is the integrated XAS (grey line, right $y$ axis) after background subtraction. (c) Normalized XMCD $\left(\mu^{+}-\mu^{-}\right)$(black solid line and yellow area) and integral XMCD (grey line, right $y$ axis). 
that has been adjusted to zero in the pre-edge 304 region and to one at the peak maximum of the 305 averaged XAS. In these units, a constant value 306 of $1.7 \mathrm{arb} . u n$. corresponding to the pre-edge in- 307 tensity has been subtracted from all the spec- 308 tra.

Fig. 2(b) shows the polarization-averaged 310 XAS $12\left(\mu^{+}+\mu^{-}\right)$with a steplike continuum 311 background. The lineshape of the $\mathrm{Eu} M_{5,4} 312$ edge XAS displays clearly divalent $\mathrm{Eu}^{2+} .{ }^{25-27}{ }_{313}$ Therefore, we exclude different integer oxida- 314 tion states. The $3: 2$ ratio of the step heights 315 (branching ratio) at the $M_{5}$ and $M_{4}$ edges re- 316 sults from the degeneracy of the $3 d_{5 / 2}$ and $3 d_{3 / 2} 317$ orbitals. This is in agreement with Eu XAS ${ }_{318}$ analysis in literature. ${ }^{27-29}$ By subtracting the 319 continuum background we separate the $M_{5,4} 320$ contributions (blue area) from the averaged 321 XAS. The integral $r$ [Fig. 2(b), right axis] of 322 the $M_{5,4}$ contributions is used for normalization ${ }_{323}$ in the sum rule analysis.

Fig. 2(c) displays the XMCD signal $\left(\mu^{+}-\mu^{-}\right) 325$ which results from the subtraction of the two 326 absorption spectra with positive and negative 327 helicity. By applying sum rule analysis to the 328 XMCD data we can approximate the orbital 329 $\left(\mu_{L}\right)$ and spin $\left(\mu_{S}\right)$ magnetic moments: ${ }^{28,30}$

$\mu_{l}=-n_{h} \frac{q}{r} \mu_{B}$

(2) 333

$\mu_{S}=-n_{h} \frac{5 p-3 q}{2 r} \mu_{B}-6\left\langle T_{z}\right\rangle \mu_{B} \approx-n_{h} \frac{5 p-3 q}{2 r} \mu_{33 B}^{334}$

(3) 336

337

where the values $p$ and $q$ [Fig. 2(c), right axis] ${ }_{338}$ describe the integrals of the XMCD over the 3 З $M_{5}$ and $M_{5,4}$ edges, respectively, $n_{h}$ identifies ${ }_{340}$ the number of holes in the $4 f$ shell (here $n_{h}={ }_{341}$ 7) and $r$ is the integral of the averaged XAS, ${ }_{342}$ mentioned above. We approximated the dipolar ${ }_{343}$ term $\left\langle T_{z}\right\rangle$ as zero corresponding to the atomic ${ }_{344}$ properties of $\mathrm{Eu}^{2+}$ in $4 f^{7}$ configuration.

Applying sum rule analysis, we confirm the 346 orbital moment $\mu_{L}$ to be zero within the error ${ }_{347}$ bar of our measurements, i.e. $\mu_{L}=\left(-0.1 \pm{ }_{348}\right.$ 0.3) $\mu_{B}$, but also allows the interpretation of a 349 small but finite value. For the spin moment $\mu_{S} 350$ we obtain $\mu_{S}=(+7.0 \pm 0.6) \mu_{B}$, which is in ${ }_{351}$ good agreement with the expectation from the 352 atomic properties of $+7.0 \mu_{B}$. We note: (1) The measured magnetic moments of the Eu ion display, strictly speaking, only the time-averaged projection of the moments along the x-ray direction. We are confident that, for a sample temperature of $5 \mathrm{~K}$, we are in the vicinity of saturation. Thus, we rule out temperaturedependent fluctuations of the calculated magnetic moment and equate them with the true saturated values. (2) Applying the sum rules for rare earths is challenging, in particular due to the uncertainties of the separation of the absorption edges and the long-range magnetic background. For more information on the origin of the errors in the sum rule analysis, see the supplementary information. In good approximation, the calculated values from the sum rule analysis imply that $\mathrm{Eu}$ is present in the halffilled $4 f^{7}$ configuration as in its bulk state. This is in agreement with the divalent $\mathrm{Eu}^{2+}$-state that we derived from the spectral lineshape of the averaged XAS.

In addition we performed XAS and XMCD measurements in a magnetic field of $B=9 \mathrm{~T}$ for sample temperatures of $T=7 \mathrm{~K}$ and $T=$ $10 \mathrm{~K}$, which results in a slightly reduced magnetization by $9 \%$ and $10 \%$, respectively, as compared to the value at $5 \mathrm{~K}$. This reduction of $\mu_{S}$ is attributed to the ensuing increased spin fluctuations with increasing temperature. For more details on the temperature dependent XAS measurements, see the supplementary information.

To investigate magnetic coupling and anisotropy of the system, in Fig. 3 we plot the field-dependent XMCD signal at the $M_{5}$ edge normalized to the pre-edge value in dependence of the magnetic field. We assume the $\mathrm{Eu}$ magnetization to be proportional to the XMCD signal. The magnetization is given in arbitrary units scaled to a value of 1.0 at $B=9$ T. While Fig. 3(a) measured at the lowest attainable temperature of $5 \mathrm{~K}$ and normal incidence $\left(\theta=0^{\circ}\right)$ displays no loop opening, Fig. 3(b) recorded at the same temperature and grazing incidence with $\theta=60^{\circ}$ shows a clear hysteresis with a coercive field of $0.2 \mathrm{~T}$ [compare inset of Fig. 3(b)]. We note that measuring the in-plane magnetization displays an 


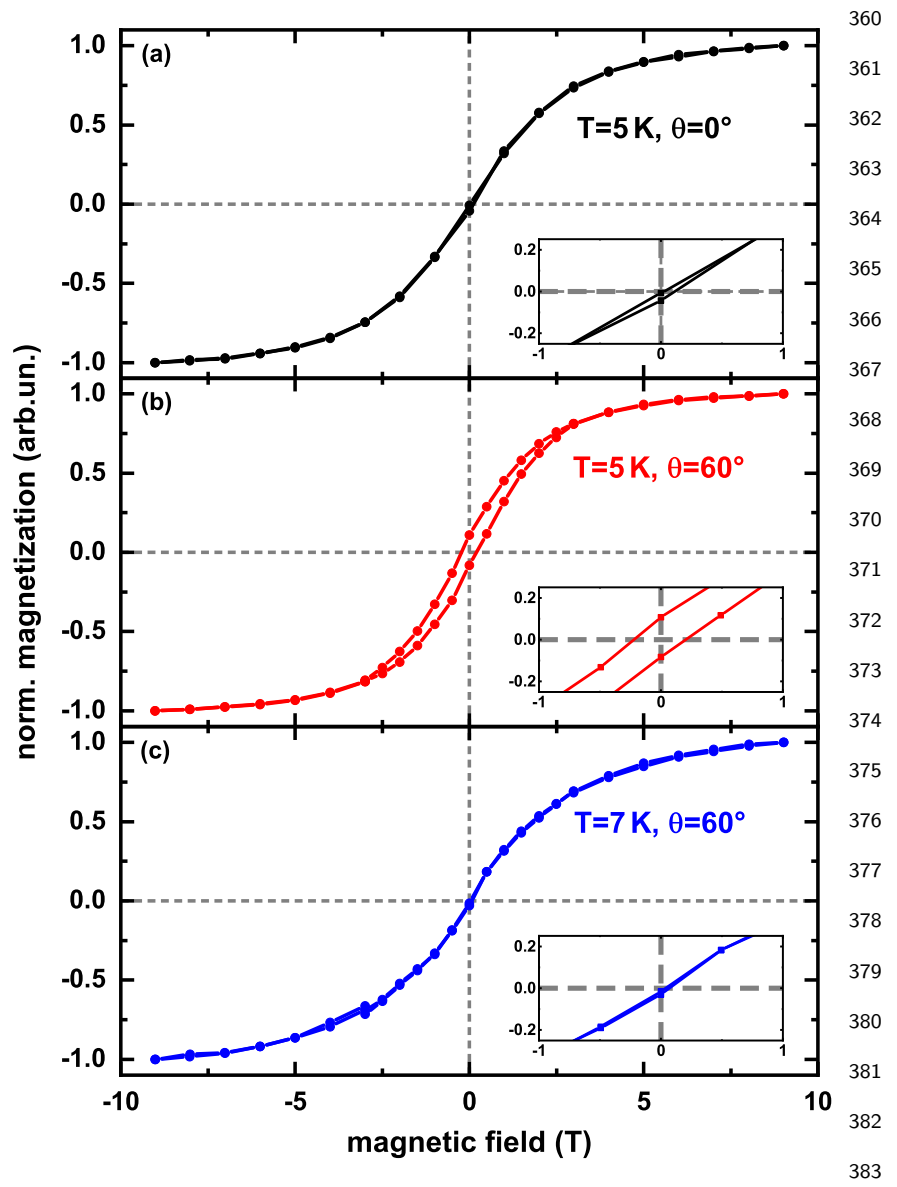

Figure 3: Field-dependent XMCD signal at the 384 Eu $M_{5}$ edge $\left(E_{h v}=1130.1 \mathrm{eV}\right)$ for $-9 \mathrm{~T} \leq B \leq 385$ $9 \mathrm{~T}$, normalized such that the field-dependent 386 XMCD signal at $B=9 \mathrm{~T}$ is 1.0 . Insets mag- 387 nify the magnetization in the range $-1 \mathrm{~T} \leq 388$ $B \leq 1 \mathrm{~T}$. (a) Magnetization for normal inci- 389 dence with $\theta=0^{\circ}$ at $5 \mathrm{~K}$. (b) Magnetization for 390 grazing incidence with $\theta=60^{\circ}$ at $5 \mathrm{~K}$. (c) Mag- 391 netization for grazing incidence with $\theta=60^{\circ}$ at 392 $7 \mathrm{~K}$.$$
364
$$

365$$
70
$$

371

372

373

374

375

377

378

379

380$$
\text { (n) }
$$$$
83
$$

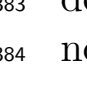

Previous calculations ${ }^{9}$ gave the single-ion anisotropy of $\mathrm{Eu}$ in EuCot to be on the order of only a few eV, consistent with our own DFT calculations. However, as will be discussed below, a much higher magnetocrystalline anisotropy of about $0.5 \mathrm{meV}$ has to be present to explain the experimental observations. In contrast, the anisotropy energy resulting from the magnetic dipolar interaction is on the order of $100 \mathrm{eV}$ due to the large moment of $7 \mu_{B}$, and, according to these calculations, would thus entirely dominate the magnetic anisotropy. More precisely, we have calculated the dipolar energy for three different orientations of magnetic moments in the EuCot nanowire carpet: (a) along the wire axis, $E_{a}=-0.0745 \mathrm{meV}$; (b) perpen- 
dicular to wire axis, but in the plane of the car- 451 pet, $E_{b}=-0.0050 \mathrm{meV}$; and (c) perpendicular ${ }_{452}$ to the plane of the carpet, $E_{c}=+0.0795 \mathrm{meV} .{ }_{453}$

This means, for normal x-ray incidence all 454 wires are magnetized in a hard direction - explaining the absence of hysteresis - while for grazing incidence, despite the random in-plane orientation of the wires, for some EuCot islands a large component of the applied magnetic field is along the easy axis. In this view, the moderate susceptibility at grazing x-ray incidence is tentatively assumed to result from the difficulty to orient the magnetic moments in EuCot wire islands that are substantially misoriented with respect to the projection of the magnetic field onto the sample plane.

We also considered alternative explanations for the magnetization loop opening that can ${ }^{45}$ be excluded as explained in the following: 1) One might speculate that ferromagnetic EuO has formed, during growth or later by oxidation from the residual gas, which certainly would give rise to a loop opening. However, (i) EuCot synthesis is efficient and performed in large Cot excess, such that we never observed any sign of metallic Eu on the sample with STM after EuCot growth, which also rules out $\mathrm{EuO}$ formation during subsequent treatment. Moreover, (ii) EuO is magnetically soft, with a coercive field of less than $100 \mathrm{mT},{ }^{32}$ and thus cannot be responsible for an opening persisting up to $2.5 \mathrm{~T}$. In addition, (iii) $\mathrm{EuO}$ has a $T_{C}=69 \mathrm{~K}$, inconsistent with our finding of $T_{C} \leq 7 \mathrm{~K}$. 2) We considered that a layer of intercalated Eu under Gr could have accidentally formed, which would be strongly ferromagnetically coupled. However, as mentioned already above, (i) with STM we never observed any sign of metallic Eu, be it adsorbed or intercalated, on the sample after EuCot growth. Moreover, (ii) intercalated $\mathrm{Eu}$ is magnetically soft as well, as discussed in Ref. 27. The large coercive field can then only be explained by a strong in-plane uniaxial anisotropy, and $\mathrm{Eu}$ in EuCot islands is the only possible explanation. Consistent with the 456 magnetic loop opening due to the presence of ${ }_{457}$ $\mathrm{Eu}$ in EuCot is the fact that the loop opening ${ }_{458}$ is subject to x-ray radiation damage when the ${ }_{459}$ sample is intentionally illuminated with higher 460 $\mathrm{x}$-ray flux, as typical for an organometallic system. For more details on the effect of radiation damage on the magnetization curves, see the supplementary information.

Figure 4: (a) $d I / d V$ point spectrum on a EuCot island. The tip was stabilized at $U=-1.0 \mathrm{~V}$ and $I=5 \mathrm{nA}$ prior to feedback loop opening. The spectrum displayed is the average over 5 subsequent spectra with the same tip at the same location of an EuCot island indicated by the blue dot in the STM topograph shown as inset. Image size $25 \times 100 \mathrm{~nm}, U=-2.0 \mathrm{~V}$, $I=66 \mathrm{pA}$. Thin vertical lines indicate positions where the $d I / d V$ signal raises over the noise level in the tunneling gap. (b) The projected density of states for $\mathrm{Eu} 5 d$ and $\mathrm{C} 2 p$ orbitals for spin up (positive) and spin down (negative).
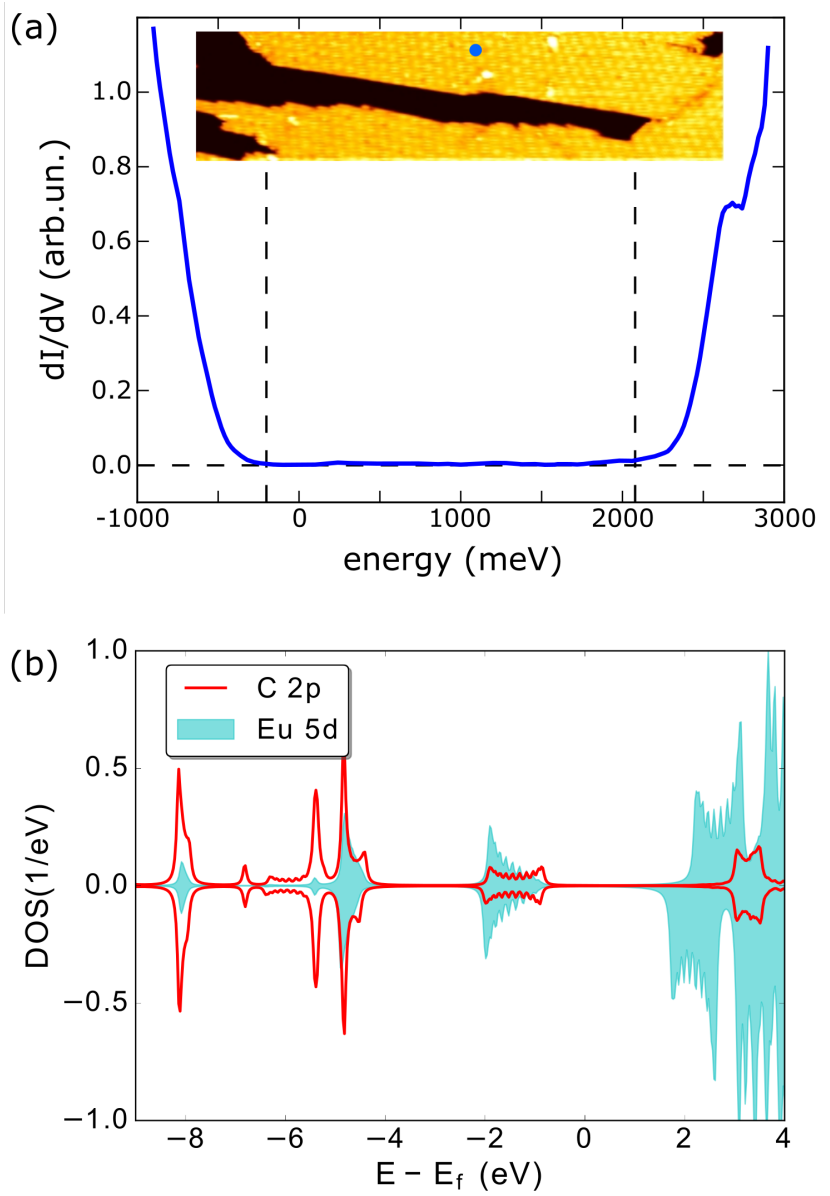

Fig. 4 provides further insight into the electronic structure of the EuCot nanowires. In Fig. 4(a) a typical STS point spectrum taken on a monolayer thick EuCot island is provided (see inset for location of spectroscopy). From the 
spectrum it is obvious that the EuCot wires are 510 wide band gap semiconductors. The electronic 511 band gap is estimated to $2.3 \mathrm{eV}$ and ranges be- 512 tween $-0.2 \mathrm{eV}$ and $2.1 \mathrm{eV}$. For this estimate 513 the band edges were identified as the locations 514 where the $d I / d V$ intensity moves just out of 515 the noise level within the gap. Note that due 516 to their localized nature, the Eu $4 f$ states are ${ }_{517}$ not expected to contribute to the tunneling cur- 518 rent. They are therefore invisible in STM and 519 may be located within the gap as measured by 520 STS. Therefore the measured band gap corre- ${ }^{521}$ sponds to the minority bandgap and is consid- 522 erably smaller than the $\approx 3 \mathrm{eV}$ calculated be- ${ }_{523}$ fore. ${ }^{15,16}$

524

The spin components of the density of states 525 (DOS) resulting from our DFT calculations are 526 shown in Fig. 4(b). As can be seen, the sys- ${ }^{527}$ tem is an insulator with a band gap of about ${ }_{528}$ $2.1 \mathrm{eV}$, which agrees rather well with the exper- ${ }_{529}$ imentally observed gap. The bandgap is de- 530 termined for our case by the minority chan- 531 nel. In Fig. 4(b) we only show the dominant 532 parts of the atom and l-projected DOS curves, 533 i.e. the $\mathrm{C} 2 p$ and $\mathrm{Eu} 5 d$ states. Note that 534 since the $4 f$ electrons are localized, we have ex- 535 cluded them from the graph. However, the $4 \mathrm{f} 536$ electron states may come into play for excita- 537 tion energies of around $2 \mathrm{eV}$, since the energy 538 difference between the di- and trivalent state 539 is about $2 \mathrm{eV}$. We conclude this based on the ${ }_{540}$ Born-Haber analysis presented in the supple- 541 mentary information. To be precise, the va- 542 lences of a bare Eu wire and a EuCot wire are 543 calculated to be divalent, with an energy gap 544 to the trivalent configuration of $1.94 \mathrm{eV}$ respec- 545 tively $2.08 \mathrm{eV}$.

Fig. 4(b) shows that there is a significant hy- 547 bridization between C 2p and Eu 5d states, es- 548 pecially for the occupied states. This shows up 549 most markedly from peaks that have common 550 large intensity of both types of orbitals. The 551 exchange splitting is largest for $\mathrm{Eu} 5 \mathrm{~d}$ states, 552 which is most noticeable for the unoccupied 553 states. This exchange splitting is induced by ${ }_{554}$ the exchange and correlation from the large spin 555 density of the $4 f$ shell, which has a net moment 556 of $7 \mu_{B}$. The induced exchange splitting of the ${ }_{557}$ $5 \mathrm{~d}$ states is found also for the occupied states, ${ }_{558}$ and for this reason a small moment of $0.05 \mu_{B}$ emerges on the Eu valence states (excluding the $7 \mu_{B}$ of the $4 f$ shell). We note here that in this kind of calculations there is also an interstitial contribution to the moment, located between the Eu atom and the Cot molecule, that has an induced magnetization that is difficult to assign to a specific atom or orbital angular momentum state. The net induced moment, summed over interstitial contribution and all atom and angular momentum projected states, is, however, vanishing, since the induced moment of $0.05 \mu_{B}$ on Eu states is compensated exactly by the induced interstitial moment and the moment projected on the Cot molecule. This results in a calculated moment of $7 \mu_{B} /$ f.u.. An integer value of the magnetic moment is a natural outcome of any magnetic insulator, since an integer number of bands of the spin-up and spin-down states are occupied.

A theoretical analysis of the dependence of the coercive field with respect to temperature finds that the coercive field decays very fast with respect to temperature, and vanishes close to the ordering temperature (compare supplementary information). This is in agreement with our observations. The Monte Carlo simulations with an exchange coupling of $1.2 \mathrm{meV}$ matched to the experimental $T_{C} \approx 6 \mathrm{~K}$ enable us to conclude that in addition to the dipolar anisotropy of about $0.15 \mathrm{meV}$ an additional and even larger magnetic anisotropy of about $0.5 \mathrm{meV}$ has to be present in order to reproduce the experimentally observed coercive field of 0.2 T. Previous ${ }^{9}$ DFT calculations yielded magnetocrystalline anisotropies of the order of $5 \mu \mathrm{eV}$. We note that the determination of the magnetocrystalline anisotropy energies is very challenging because of the smallness of the values, and we have not attempted to explain the estimated magnetic anisotropy from the DFT calculations. Furthermore, we cannot exclude the presence of other anisotropies in the system, e.g. symmetric anisotropy exchange, ${ }^{33}$ that are not included in our model, or effects stemming from the influence of the substrate.

Concluding, we have shown experimentally through a combination of XMCD and STS that EuCot is a ferromagnetic insulator. The size 
Acknowledgement We are grateful to ${ }^{593}$ Samara Keshavarz for performimg the DFT cal- ${ }^{594}$ culation and to Dr. Yaroslav Kvashnin for valu- ${ }^{595}$ able discussions. We thank the European Syn- ${ }^{596}$ chrotron Radiation Facility (ESRF) for beam- ${ }^{597}$ time allocation (experiment HC-2698) and the ${ }_{598}$ beamline ID32 staff for the kind support. ${ }_{599}$ O.E. acknowledges support from eSSENCE, the ${ }_{600}^{599}$ Swedish Research Council, the KAW foundation (projects 2013.0020 and 2012.0031) and the 601 Foundation for Strategic Research. H.W. and 602 T.M acknowledge support from DFG through 603 projects WE 2623/17-1, and MI 581/23-1. 604 H.W. and N.R. acknowledge funding by the 605 Deutsche Forschungsgemeinschaft (DFG, Ger- 606 man Research Foundation) - Projektnummer 607 278162697 - SFB 1242. S.K. and F.H. acknowl- 608 edge financial support through the Institutional Strategy of the University of Cologne within ${ }^{609}$ the German Excellence Initiative. L.M.A., ${ }^{610}$ M.B. and W.K. thank the BMBF for finan- ${ }^{611}$ cial support (no. 05K13KEA "VEKMAG"). ${ }^{612}$ L.M.A. acknowledges CAPES for funding (no. ${ }^{613}$ 9469/13-3). duced by density functional theory calculations. 581 We have analyzed the measured results using 582 an effective spin-Hamiltonian that contains in- 583 teratomic exchange, dipolar energies, magnetic 584 anisotropy and a Zeeman term. Measurements 585 are reproduced from a model where the inter- 586 atomic exchange is of order meV, the magnetic anisotropy is roughly half of the exchange and dipolar energy is roughly one order of magni- ${ }^{587}$ tude smaller that the exchange interaction. We are confident that the finding of ferromagnetic ${ }^{588}$ ordering in an experimentally well-accessible, ${ }^{589}$ surface-supported, organometallic system will ${ }_{590}$ provide new inspiration to the field of molec- ${ }_{591}$ ular spintronics.

592

of the bandgap from experiment is well repro- 580 
Sanyal, B.; Piantek, M.; Srivastava, P. 667 et al. Tailoring the Nature of Mag- 668 netic Coupling of Fe-Porphyrin Molecules to Ferromagnetic Substrates. Phys. Rev. ${ }^{669}$ Lett. 2009, 102, 047202.

670 671

(8) Nakajima, A.; Kaya, K. A Novel Network 672 Structure of Organometallic Clusters in 673 the Gas Phase. J. Phys. Chem. A 2000, 104, 176-191.

(9) Atodiresei, N.; Dederichs, P. H.; 676 Mokrousov, Y.; Bergqvist, L.; 677 Bihlmayer, G.; Blügel, S. Controlling 678 the Magnetization Direction in Molecules 679 via Their Oxidation State. Phys. Rev. Lett. 2008, 100, 117207.

(10) Hosoya, N.; Takegami, R.; Suzumura, J.; 682 Yada, K.; Miyajima, K.; Mitsui, M.; 683 Knickelbein, M.; Yabushita, S.; Naka- 684 jima, A. Formation and Electronic 685 Structures of Organoeuropium Sandwich 686 Nanowires. J. Phys. Chem. A 2014, 118, 8298-8308.

(11) Tsuji, T.; Hosoya, N.; Fukazawa, S.; 689 Sugiyama, R.; Iwasa, T.; Tsunoyama, H.; 690 Hamaki, H.; Tokitoh, N.; Nakajima, A. 691 Liquid-Phase Synthesis of Multidecker 692 Organoeuropium Sandwich Complexes 693 and Their Physical Properties. J. Phys. ${ }^{694}$ Chem. C 2014, 118, 5896-5907.

(12) Huttmann, F.; Schleheck, N.; Atodire- 696 sei, N.; Michely, T. On-Surface Synthe- 697 sis of Sandwich Molecular Nanowires on 698 Graphene. J. Am. Chem. Soc. 2017, 139, 9895-9900.

(13) Miyajima, K.; Yabushita, S.; Knickel- 701 bein, M.; Nakajima, A. Stern-Gerlach Experiments of One-Dimensional Metal- ${ }^{702}$ Benzene Sandwich Clusters: $\mathrm{M}_{n}\left(\mathrm{C}_{6} \mathrm{H}_{6}\right)_{m}{ }^{703}$ $\left(\mathrm{M}=\mathrm{Al}, \mathrm{Sc}, \mathrm{Ti}\right.$, and V). J. Am. Chem. ${ }^{704}$ Soc. 2007, 129, 8473-8480.

(14) Zhang, X.; Ng, M.-F.; Wang, Y.; Wang, J.; ${ }^{706}$ Yang, S.-W. Theoretical Studies on Struc- ${ }^{707}$ tural, Magnetic, and Spintronic Charac- ${ }^{708}$ teristics of Sandwiched $\operatorname{Eu}_{n} \operatorname{Cot}_{n+1}\left(n={ }^{709}\right.$
1-4) Clusters. ACS Nano 2009, 3, 25152522 .

(15) Xu, K.; Huang, J.; Lei, S.; Su, H.; Boey, F.; Li, Q.; Yang, J. Efficient organometallic spin filter based on Europium-cyclooctatetraene wire. $J$. Chem. Phys. 2009, 131, 104704.

(16) Yao, X.; Yuan, S.; Wang, J. Theoretical Studies of Sandwich Molecular Wires with Europium and Boratacyclooctatetraene Ligand and the Structure on a $\mathrm{H}$ Ge(001)-2x1 Surface. J. Phys. Chem. C 2016, 120, 7088-7093.

(17) van Gastel, R.; N'Diaye, A.; Wall, D.; Coraux, J.; Busse, C.; Buckanie, N.; Meyer zu Heringdorf, F.-J.; Horn von Hoegen, M.; Michely, T.; Poelsema, B. Selecting a single orientation for millimeter sized graphene sheets. Appl. Phys. Lett. 2009, 95, 121901.

(18) Wills, J.; Alouani, M.; Andersson, P.; Delin, A.; Eriksson, O.; Grechnev, A. FullPotential Electronic Structure Method, Energy and Force Calculations with Density Functional and Dynamical Mean Field Theory; Springer Series in Solid-State Sciences; Springer-Verlag Berlin Heidelberg, 2010; Vol. 167.

(19) Binder, K.; Heermann, D. Monte Carlo Simulation in Statistical Physics; An Introduction; Springer-Verlag Berlin Heidelberg, 2010; Vol. 5.

(20) Skubic, B. Spin Dynamics and Magnetic Multilayers; Dissertation: Uppsala University, 2007.

(21) Nolting, W. Theoretical Physics 8, Statistical physics; Springer International Publishing, 2018.

(22) Uppsala University, Department of Physics and Astronomy, Uppsala Atomistic Spin Dynamics code. http://physics.uu.se/uppasd, Last accessed on 2018-11-06. 
(23) Eriksson, O.; Bergman, A.; Bergqvist, L.; 754 Hellsvik, J. Atomistic Spin Dynamics: 755 Foundations and Applications; Oxford 756 University Press, 2017.

(24) Kurikawa, T.; Negishi, Y.; Hayakawa, F.; 758 Nagao, S.; Miyajima, K.; Nakajima, A.; 759 Kaya, K. Multiple-Decker Sandwich Com- 760 plexes of Lanthanide-1,3,5,7-Cycloocta- 761 tetraene $\left[\operatorname{Ln}_{n}\left(\mathrm{C}_{8} \mathrm{H}_{8}\right)_{m}\right](\mathrm{Ln}=\mathrm{Ce}, \mathrm{Nd}, \mathrm{Eu}, 762$ $\mathrm{Ho}$, and $\mathrm{Yb}$ ); Localized Ionic Bonding 763 Structure. J. Am. Chem. Soc. 1998, 120, 11766-11772.

(25) Thole, B.; van der Laan, G.; Fuggle, J.; 766 Sawatzky, G.; Karnatak, R.; Esteva, J.- 767 M. 3d x-ray-absorption lines and the $3 d^{9} 4 f^{n+1}$ multiplets of the lanthanides. Phys. Rev. B 1985, 32, 5107.

(26) Förster, D.; Klinkhammer, J.; Busse, C.; Altendorf, S.; Michely, T. Epitaxial europium oxide on $\mathrm{Ni}(100)$ with singlecrystal quality. Phys. Rev. B 2011, 83, 045424.

(27) Schumacher, S.; Huttmann, F.; Petrović, M.; Witt, C.; Förster, D.; VoVan, C.; Coraux, J.; Martínez-Galera, A.; Sessi, V.; Vergara, I. et al. Europium underneath graphene on $\operatorname{Ir}(111)$ : Intercalation mechanism, magnetism, and band structure. Phys. Rev. B 2014, 90, 235437.

(28) Carra, P.; Thole, B.; Altarelli, M.; Wang, X. X-ray circular dichroism and local magnetic fields. Phys. Rev. Lett. 1993, 70, 694 .

(29) Kachkanov, V.; Wallace, M.; van der Laan, G.; Dhesi, S.; Cavill, S.; Fujiwara, Y.; O'Donnell, K. Induced magnetic moment of Eu3+ ions in GaN. Sci. Rep. 2012, 2, 969 .

(30) Thole, B.; Carra, P.; Sette, F.; van der Laan, G. X-ray circular dichroism as a probe of orbital magnetization. Phys. Rev. Lett. 1992, 68, 1943.

(31) Lisi, S.; Gargiani, P.; Scardamaglia, M.; Brookes, N.; Sessi, V.; Mariani, C.;
Betti, M. Graphene-Induced Magnetic Anisotropy of a Two-Dimensional Iron Phthalocyanine Network. J. Phys. Chem. Lett. 2015, 6, 1690-1695.

(32) Klinkhammer, J.; Förster, D.; Schumacher, S.; Oepen, H.; Michely, T.; Busse, C. Structure and magnetic properties of ultra thin textured $\mathrm{EuO}$ films on graphene. Appl. Phys. Lett. 2013, 103, 131601.

(33) Udvardi, L.; Szunyogh, L.; Palotas, K.; Weinberger, P. First-principles relativistic study of spin waves in thin magnetic films. Phys. Rev. B 2003, 68, 104436. 